\title{
Synchronization of Hamiltonian motion and dissipative effects in optical lattices: Evidence for a stochastic resonance
}

\author{
Laurent Sanchez-Palencid* and Gilbert Grynberg \\ Laboratoire Kastler Brossel, Département de Physique de l'Ecole Normale Supérieure, \\ 24, rue Lhomond, 75231, Paris Cedex 05, France.
}

(Dated: October 27, 2018)

\begin{abstract}
We theoretically study the influence of the noise strength on the excitation of the Brillouin propagation modes in a dissipative optical lattice. We show that the excitation has a resonant behavior for a specific amount of noise corresponding to the precise synchronization of the Hamiltonian motion on the optical potential surfaces and the dissipative effects associated with optical pumping in the lattice. This corresponds to the phenomenon of stochastic resonance. Our results are obtained by numerical simulations and correspond to the analysis of microscopic quantities (atomic spatial distributions) as well as macroscopic quantities (enhancement of spatial diffusion and pump-probe spectra). We also present a simple analytical model in excellent agreement with the simulations.

PACS numbers: 05.45.-a,05.60.-k,32.80.Pj
\end{abstract}

\section{INTRODUCTION}

During the past decades, the rapid development of the experimental cooling and trapping methods for neutral particles has delivered a remarkable mastery of accurate measurement procedures of the dynamical and spectral properties of the atoms [1]. Cold atomic samples thus constitute privileged media for the study of various physical phenomena such as the properties of degenerate quantum gases of both bosons [2] and fermions [3], or nonlinear mechanical effects [4]. Among the cooling schemes, the Sisyphus mechanism [5] distinguishes by the peculiarity that it leads not only to very cold atomic samples but also to the trapping of the atoms in spatially periodic potential wells $[6,7,8]$ by pure optical means. This creates a periodic array of atoms bound by light, the so-called optical lattices. Moreover, the trapping potentials can be easily designed in a large variety of different topographies in one, two or three dimensions [9, 10] and this provides the possibility of modeling different situations. The cooling mechanism itself results from the intercombination of Hamiltonian motion and dissipative processes and is thus particularly well suited for the study of the dynamics of particles trapped in periodic structures in the presence of noise.

In the Sisyphus scheme, a two level atom with Zeeman degeneracy interacts with a laser field with a gradient of the polarization. The light induces a spatially periodic modulation of the optical potentials (light shifts) of the different Zeeman sublevels and also random quantum jumps between these sublevels (optical pumping). The cooling mechanism results from successive cycles of Hamiltonian motion on the optical potential surfaces followed by dissipative processes associated to the random jumps in such a way that a moving atom on average

*Electronic address:

URL: http://www.1kb.ens.fr/ lsanchez climbs up potential hills before it is optically pumped into a lower lying potential surface [5]. The kinetic energy is thus converted into potential energy which is subsequently carried away by a spontaneously emitted photon, thereby reducing the total atomic energy. Because the steady-state kinetic energy is generally lower than the potential depth, the particles get trapped in the potential wells. The typical evolution times corresponding to the Hamiltonian motion on the one hand and to the dissipative processes on the other hand can vary independently with the lattice parameters and for usual experimental conditions have roughly the same order of magnitude. This leads to distinguish two dynamical regimes (the jumping regime where dissipative effects dominate and the oscillating regime where Hamiltonian motion dominates). This makes particularly difficult to perform analytical calculations in the whole accessible range of parameters [11].

By contrast, the intermediate regime offers the possibility of studying unusual and fascinating atomic behaviors combining Hamiltonian motion and dissipative effects. In particular, it can be considered as models for the transport of particles trapped in periodic structures and in the presence of noise, which is of particular interest for systems where the dynamics is dominated by noise such as in many biological systems [12. For example, an atomic ratchet has been realized in a variant of the configuration considered in this paper 13] and the phenomenon of directed diffusion has been observed [14] in a one-dimensional setup.

In a recent publication [15], we identified a resonant behavior of the stimulated transport (Brillouin propagation modes) of atoms versus the noise strength, i.e. versus the rate of optical pumping, and we interpreted it in terms of a stochastic resonance (SR). This phenomenon, corresponding to a situation where Hamiltonian motion and noise-induced effects work together to induce a resonant response of a system to a weak external stimulation, has been widely studied in various theoretical models [16]. However, no direct evidence for SR in dissipative optical 
lattices has been given so far. The aim of this paper is to theoretically analyze the influence of the level of noise on the efficiency of the excitation process of the Brillouin propagation modes in a dissipative optical lattice. We show that the excitation is resonant for a specific amount of noise corresponding precisely to the synchronization of the hamitonian motion and of the dissipative effects. Our results are mainly obtained by means of numerical simulations.

Our paper is organized as follows. In section III we present the main features of the dynamics of atoms cooled and trapped in a dissipative optical lattice. We discuss the peculiarities of Hamiltonian motion and dissipative processes and we describe the Brillouin propagation modes. In the next two sections, we show that the excitation of the propagation modes is resonant for a given amount of noise and that this is compatible with the phenomenon of SR: (i) in section [II] we analyze the resonant enhancement of the spatial diffusion of the atomic cloud and we show that the position of the resonance precisely corresponds to the synchronization of the Hamiltonian motion and the dissipative processes; (ii) in section IV] we present further evidences for the phenomenon of SR by considering the microscopic behavior of the propagating atoms. We also show that SR can be observed in the amplitude of the Brillouin resonance that appears in well-mastered nonlinear spectroscopy experiments. Finally, we summarize our results in section $\nabla$

\section{HAMILTONIAN MOTION AND DISSIPATIVE PROCESSES IN OPTICAL LATTICES}

\section{A. Dynamics of cold atoms in an optical lattice}

Consider a sample of atoms interacting with a laser field with a frequency slightly red detuned with respect to a $J \rightarrow J+1$ atomic transition. The atoms experience a set of optical potentials corresponding to the light shifts of the various Zeeman sublevels of the atomic ground state and thus depending on the internal state. The topography of the potential surfaces depends on the interference pattern of the laser fields and are spatially periodic with periods of the order of the laser wavelength. In this paper we study the so-called $3 \mathrm{D}$-lin $\perp$ lin configuration [17]. This is obtained from the standard 1D-lin $\perp$ lin configuration [5] by symmetrically splitting each of the two laser beams into two parts at an angle $\theta$ with the $(O z)$ axis in the $(O x z)$ and $(O y z)$ planes respectively. The resulting configuration consists of two pairs of laser beams in the $(O x z)$ plane and in the $(O y z)$ plane, respectively with orthogonal linear polarizations as depicted in Fig. [1 In this situation, the potential surfaces are made of regularly displaced wells (see Fig. (2).

Moreover, for a near resonant laser field, the optical pumping cycles transfer the atoms from a given sublevel to another one with a rate depending on the atomic posi-

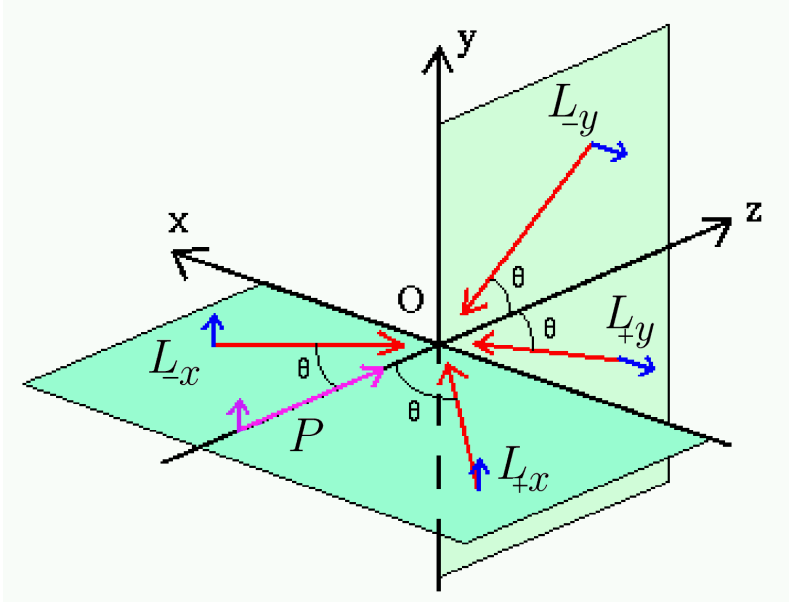

FIG. 1: 3D-lin $\perp$ lin laser configuration: the interference pattern of the four laser beams $L_{ \pm \mu}$ (with $\mu \in\{x, y\}$ ), with linear polarizations arranged as in the figure, creates the optical lattice. A probe laser beam $(P)$ along the $z$ - axis is also added for excitation of propagation modes (see section IIB) and nonlinear spectroscopy (see section IVB).

tion. More precisely, the optical pumping tends to populate the lower potential surfaces thus providing the cooling of the moving atoms via the Sisyphus effect [5, 11]. The resulting mean atomic kinetic energy is lower than the potential barrier for escaping from the potential wells, so that the atoms get trapped and form a crystal-like lattice bound by light.

After the cooling stage, the atomic dynamics is composed of two parts: the Hamiltonian motion and dissipative effects. First, the hamitonian motion consists in periodic oscillations around the bottom of potential wells. The principal directions of the oscillations correspond to the coordinates axes $x, y$ and $z$ with a frequency $\Omega_{\mu} / 2 \pi$ along the $\mu$ - axis. In particular, in the $3 \mathrm{D}$-lin $\perp$ lin configuration and for a $J_{\mathrm{g}}=1 / 2 \rightarrow J_{\mathrm{e}}=3 / 2$ atomic transition, one finds

$$
\Omega_{x}=4 \sin (\theta) \sqrt{\left|\Delta_{0}^{\prime}\right| \omega_{\mathrm{r}}}
$$

where $\theta$ is half the angle between the quasi-copropagating laser beams (see Fig. 10 and $\Delta_{0}^{\prime}$ is the light shift per lattice beam and for a Clebsch-Gordan coefficient of 1 [10]. $\omega_{\mathrm{r}}=\frac{\hbar k^{2}}{2 M}$, with $k$, the laser wavenumber and $M$ the mass of one atom, denotes the recoil frequency. In the following, we only consider the case $\theta=30^{\circ}$. Second, the dissipative processes correspond to random quantum jumps between the various potential surfaces that modify instantaneously the atomic energy. For the same conditions as for Eq. (11) and in the semi-classical approximation, the optical pumping rate from the internal state $|q, \pm\rangle$ to the state $|g, \mp\rangle$ averaged over position, reads [18]

$$
\gamma_{ \pm, \mp} \simeq \frac{4 \Gamma_{0}^{\prime}}{9}\left\{\overline{\cos ^{2}(2 \pi \hat{x})+\cos ^{2}(2 \pi \hat{y})}\right\}
$$


where $\Gamma_{0}^{\prime}$ is the scattering rate of photons per lattice beam and for a Clebsch-Gordan coefficient of $1, \hat{x}$ and $\hat{y}$ denotes the atomic position along the $x$ - and $y$ - directions in units of the lattice spacing in the corresponding direction and $\overline{(.)}$ denotes the uniform spatial average value [35].

As pointed out in section \ the relative importance of Hamiltonian evolution and dissipative effects depends on the lattice parameters. In the jumping regime $\left(\Gamma_{0}^{\prime} \gg \Omega_{x}\right)$, the dissipative effects dominate the dynamics so that an atom encounters several optical pumping cycles during a single oscillation in a potential well. On the contrary, in the oscillating regime $\left(\Omega_{x} \gg \Gamma_{0}^{\prime}\right)$, the dynamics is dominated by the Hamiltonian motion and an atom makes several oscillations before being optically pumped. Note that in our configuration, $\Delta_{0}^{\prime} \propto I_{\mathrm{L}} / \Delta$ and $\Gamma_{0}^{\prime} \propto I_{\mathrm{L}} / \Delta^{2}$, where $I_{\mathrm{L}}$ is the intensity of one laser beam and $\Delta$ is the detuning between the laser and the Bohr frequencies of the atomic transition, so that the typical times of both Hamiltonian motion $\left(2 \pi / \Omega_{x}\right)$ and of the dissipative effects $\left(1 / \gamma_{ \pm, \mp}\right)$ can vary independently in a large range.

Finally, because a strong trapping site in the potential surface for a given internal state (for example $x=y=z=0$ for $|-\rangle$ ) corresponds to a non-trapping site in the potential surface for an other internal state (see Fig. 21),$\gamma_{ \pm, \mp}$ is approximately the escape rate from a trapping site. Note that the macroscopic manifestation of the escapes from trapping sites consists in spatial diffusion of the atomic cloud in the lattice [19].

\section{B. Brillouin Propagation modes}

One can excite atomic propagation modes in the optical lattice by adding a laser beam $(P)$ detuned by $\delta$ from the lattice beams and aligned with the symmetry axis $z$ as shown in Fig. 10 15, 20]. This laser is usually referred to as the probe beam because it was first introduced in nonlinear spectroscopy experiments in optical lattices ( 7, 21], see also section [VB). For an atom, a propagation mode in a given direction consists in making a half oscillation in a potential well followed by an optical pumping cycle that changes its internal state, then in making a new half oscillation in the neighboring well, undergoing an optical pumping cycle and so on, as shown in Fig. 2]

The interference between the probe laser beam and the lattice beams induces a moving spatial modulation of the potential surfaces. For the configuration considered here, there are two modulations symmetric with respect to $z$ along the directions $\vec{u}^{ \pm}=\overrightarrow{\Delta k}^{ \pm} /|\overrightarrow{\Delta k} \pm|$ where $\overrightarrow{\Delta k}^{ \pm}$is the difference between the wavevectors of $L_{ \pm x}$ and $P$ and that lies in the $(O x z)$ plane. Note that there are also two moving potential modulations in the $(O y z)$ plane. However, we do not consider them because they are of a very different kind and in particular, they excite the propagation modes in the $(O y z)$ plane only for a value

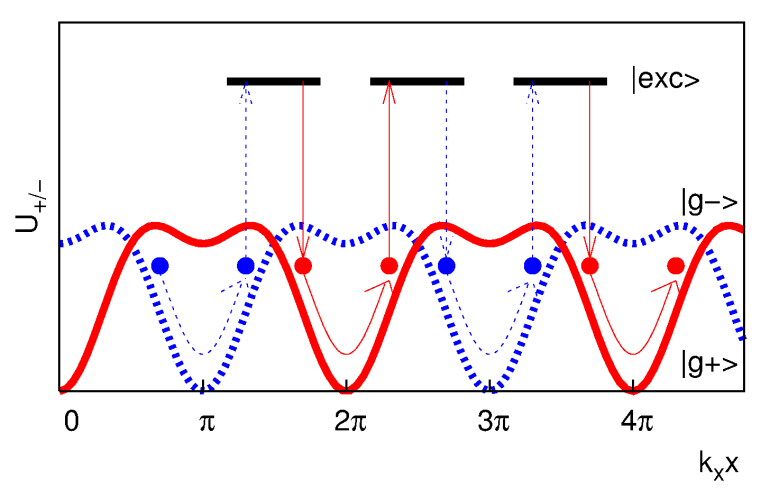

FIG. 2: Cut along a given direction $(x)$ of the optical potentials $U_{ \pm}$for a $J_{\mathrm{g}}=1 / 2 \rightarrow J_{\mathrm{e}}=3 / 2$ atomic transition. The propagation mode along $+x$ is also represented. Similar modes exist along $-x, \pm y$ and $\pm z$.

of $\delta$ different from the one considered in the following (see Eq. (3)). The potential modulations are periodic in space with a period $\lambda_{\text {mod }}=2 \pi /\left|\overrightarrow{\Delta k}^{ \pm}\right|$and move along $\vec{u}^{ \pm}$at a velocity $v_{\text {mod }}=\delta /\left|\overrightarrow{\Delta k^{ \pm}}\right|$. In particular, in the $x$ - direction, the modulations move along $+x$ and $-x$ at a velocity $v_{x}=\delta /\left|\overrightarrow{\Delta k^{ \pm}} \cdot \overrightarrow{e_{x}}\right|$. Because the atoms tend to populate the potential wells of the moving modulation, the two modulations dragg the atoms along $+x$ and $-x$ at a velocity $v_{\text {mod }}$. Hence, a resonant excitation (Brillouin resonance) of the propagation mode is obtained when the phase velocity $v_{\text {mod }}$ of the driving modulations is equal to the natural velocity $v_{x}$ of an atom along the mode in the lattice. $v_{x}$ corresponds (see Fig. (2) for an atom to travel over one half spatial period $\left(\pi / k_{x}\right)$ in half a temporal oscillating period $\pi / \Omega_{x}$, so that $v_{x}=\Omega_{x} / k_{x}$. In the $3 \mathrm{D}$-lin $\perp$ lin lattice, one finds that the Brillouin resonance for the propagation modes along the $x$ - direction corresponds to

$$
\delta= \pm \Omega_{x} .
$$

In the following, we consider situations where the propagation modes in the $x$ - direction are resonantly excited versus $\delta$. The atomic cloud thus separates into three modes. The first one corresponds to atoms trapped in the potential wells of the unmoving lattice and that are not sensitive to the moving potential modulations whereas the other two correspond to atoms moving along the propagation modes at a velocity $v_{\text {mod }}$ towards $+x$ or $-x$. These modes are not independent. There are population transfers between the modes, mainly due to nonHamiltonian forces encountered by an atom which are related to random optical pumping cycles and fluorescence cycles.

\section{Numerical simulations}

Our theoretical analysis is supported by numerical simulations. These consist in a Monte-Carlo integration of 
the master equation for the dynamics of an atom interacting with a laser field and the vacuum modes in the semi-classical approximation [18, 19]. This approximation is justified in the range of parameters that we consider here because of the strong localization of the atomic wavefunctions [11]. We consider a theoretical transition $J_{\mathrm{g}}=1 / 2 \rightarrow J_{\mathrm{e}}=3 / 2$ which gives good qualitative results and for which analytical calculations can be performed [5].

In the semi-classical approximation of the laser cooling theory, the master equation can be written as a FokkerPlanck equation (FPE) [18, 19, 22] where the external degrees of freedom of the atoms are treated as classical variables. This is obtained from the Wigner transform [23] of the full quantum master equation for external as well as internal degrees of freedom under the assumption of a momentum distribution which is much broader than a single photon momentum, $\Delta P \gg \hbar k$. The FPE for the populations $\Pi_{ \pm}(r, p, t)$ of $| \pm\rangle$ reads

$$
\begin{aligned}
& {\left[\partial_{t}+\frac{p_{i}}{M} \partial_{i}-\left(\partial_{i} U_{ \pm}\right) \partial_{p_{i}}\right] \Pi_{ \pm}=} \\
& \quad \gamma_{\mp, \pm} \Pi_{\mp}-\gamma_{ \pm, \mp} \Pi_{ \pm} \\
& \quad-F_{ \pm \pm}^{i} \partial_{p_{i}} \Pi_{ \pm}-F_{\mp \pm}^{i} \partial_{p_{i}} \Pi_{\mp} \\
& \quad+D_{ \pm \pm}^{i j} \partial_{p_{i}} \partial_{p_{j}} \Pi_{ \pm}+D_{\mp \pm}^{i j} \partial_{p_{i}} \partial_{p_{j}} \Pi_{\mp} .
\end{aligned}
$$

Here $i, j=x, y, z$ and summation over $i$ and $j$ is assumed. In this equation, $\gamma_{ \pm, \mp}$ is the jumping rate from the Zeeman sublevel $| \pm\rangle$ to the sublevel $|\mp\rangle, F_{ \pm \pm}^{i}$ represents the radiation pressure force and $D_{ \pm \pm}^{i j}$ the momentum diffusion matrix for atoms in the internal state $| \pm\rangle . F_{ \pm \mp}^{i}$ and $D_{ \pm \mp}^{i j}$ are the corresponding quantities associated with jumps between different internal states [24]. Note that all these coefficients are laser field depend. In our case the laser field is made of the contributions of the lattice beams $L_{ \pm \mu}$ and of the probe beam $P$. A numerical solution of the FPE can be obtained by averaging over many realizations of the corresponding Langevin equations [19, 25].

For the sake of simplicity and to save computation time, we restricted the atomic motion into two dimensions of space, in the plane $y=0$. That way, we consider the motion along $x$ (direction of the stimulated propagation modes) and also along $z$ to take into account the possible escapes in the orthogonal direction. As pointed out in the preceeding section, we only consider cases where only the propagation modes in the $(O x z)$ are excited. We therefore expect our results to differ from 3D situations only by scaling factors due to the atomic spatial distribution in the $y$ direction (see also the discussion at the end of section (IIIB).

\section{MACROSCOPIC MANIFESTATION OF STOCHASTIC RESONANCE}

\section{A. Resonant enhancement of spatial diffusion}

The excitation of the propagation modes drives one part of the atomic cloud along $+x$ and another part along $-x$. The spatial diffusion in the specific direction $x$ thus results enhanced whereas the spatial diffusion in the orthogonal directions is almost unchanged [15]. In order to optimize this phenomenon for a given set of lattice parameters, we tune the probe laser frequency to the Brillouin resonance according to Eq. (3). Because the propagation process of an atom along a mode involves both Hamiltonian motion and dissipative processes, it is clear that changing the strength of one with respect to the other should modify the efficiency of the driving. We plot in Fig. 3 the spatial diffusion coefficient in the lattice along the driving direction $\left(D_{x}\right)$ and in the orthogonal direction $\left(D_{z}\right)$ as a function of the noise strength (proportional to the optical pumping rate $\Gamma_{0}^{\prime}$ ).

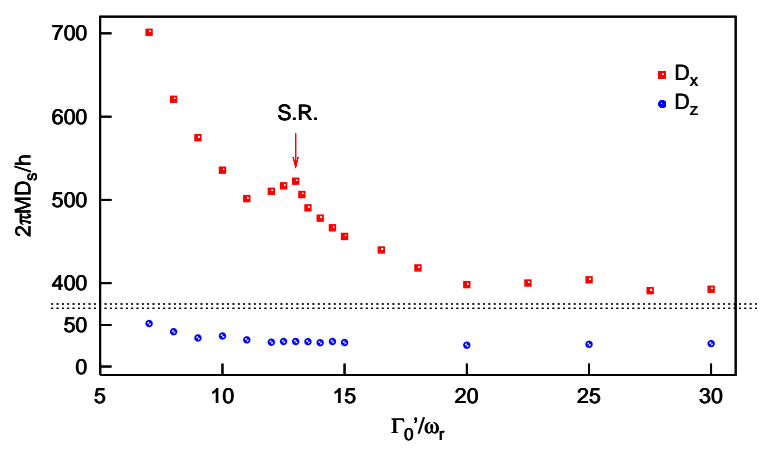

FIG. 3: Numerical results for the spatial diffusion coefficients in the $x$ - and $z$-directions as a function of the optical pumping rate, for a given depth of the optical potential. Parameters for the calculations are: $\Delta_{0}^{\prime}=-200 \omega_{\mathrm{r}}$ and $\theta=30^{\circ}$.

Generically, $D_{\mu}$ shows a monotonous decrease versus $\Gamma_{0}^{\prime}$. This behavior is in agreement with a simple model of random walk [26]: an atom is trapped in a potential well with a lifetime of the order of $\tau_{\text {esc }} \sim 1 / \Gamma_{0}^{\prime}$ and during a jump it travels at a typical velocity of $\bar{v}=\sqrt{2 E_{\mathrm{K}} / M}$ (where $E_{\mathrm{K}}$ is the kinetic energy of the cloud and $M$ is the mass of one atom) for a typical time $\tau_{\text {flight }} \sim 1 / \Gamma_{0}^{\prime}$. Because for Sisyphus cooling, $E_{\mathrm{K}} \propto \hbar\left|\Delta_{0}^{\prime}\right|$ [5], the spatial diffusion coefficient scales as $D_{\mu} \sim \frac{\hbar}{M} \frac{\left|\Delta_{0}^{\prime}\right|}{\Gamma_{0}^{\prime}}$ [19]. In addition to the global behavior versus $\Gamma_{0}^{\prime}$, we find that $D_{x}$ displays a clear narrow peak for a specific value of $\Gamma_{0}^{\prime}$ $\left(\Gamma_{0}^{\prime} \simeq 13 \omega_{\mathrm{r}}\right.$ in Fig. 3) whereas no peak is observed for the spatial diffusion coefficient along the orthogonal $(z-)$ direction. This corresponds to a resonant enhancement of the spatial diffusion along the driving direction and can be interpreted as a resonant excitation of the Brillouin propagation modes for a given amount of noise. This point will be demonstrated in detail in section IVA. 


\section{B. Characterization of stochastic resonance}

In order to characterize the specific influence of the propagation modes to the spatial diffusion in the $x$ - direction, we introduce the enhancement factor of the spatial diffusion coefficient along $x$ with a probe beam at Brillouin resonance (see Eq. (3) ) $D_{x}$ compared to far from Brillouin resonance $D_{x}^{0}$ :

$$
\xi=\frac{D_{x}-D_{x}^{0}}{D_{x}^{0}}
$$

Here, $D_{x}^{0}$ is typically calculated for $\delta \simeq 100 \Omega_{x}$. In Fig. 4 we plot the enhancement factor $\xi$ versus the noise strength $\Gamma_{0}^{\prime}$ for a fixed value of the potential depth $\Delta_{0}^{\prime}$ and for various depths of the driving modulation induced by the probe beam.

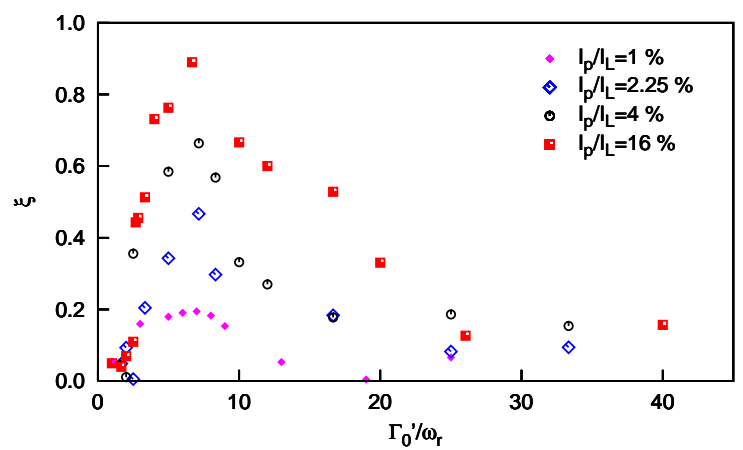

FIG. 4: Numerical results for the enhancement factor $\xi$ as a function of the optical pumping rate, for a given depth of the optical potential. The four data sets correspond to different intensities of the probe beam $I_{\mathrm{P}}$ in units of the intensity of one lattice beam $I_{\mathrm{L}}$, i.e. different depths of the moving potential modulation. Parameters for the calculations are: $\Delta_{0}^{\prime}=-50 \omega_{\mathrm{r}}$ and $\theta=30^{\circ}$.

The results of Fig. 4 show that $\xi$ is a nonmonotonic function of the amount of noise $\Gamma_{0}^{\prime}$ in the lattice. For low noise strength, $\xi$ is an increasing function of $\Gamma_{0}^{\prime}$, then $\xi$ reaches a maximum and finally decreases for high values of the noise strength $\Gamma_{0}^{\prime}$. Hence, there is a specific value of the noise strength for which the propagation modes are resonantly excited. Such a property is interpreted as a SR and corresponds to the synchronization of Hamiltonian motion and dissipative processes [16]. We checked that the peak for $\xi$ precisely corresponds to the peak for $D_{x}$ and we conclude that the resonant enhancement of spatial diffusion is due to a resonant excitation of the propagation modes in the $x$ - direction.

In order to understand more precisely the phenomenon in our system, consider again the propagation process of an atom in a Brillouin mode (section IIB and Fig. 21). Such a process clearly works better when an optical pumping cycle occurs each time the atom gets close to a crossing point between the potential curves $U_{+}$and $U_{-}$. Taking into account the velocity of the atoms in the propagation modes $v_{x}=\Omega_{x} / k_{x}$, this occurs periodically in time every $\pi / \Omega_{x}$. Besides, the typical time between two subsequent optical pumping cycles is $1 / \gamma_{ \pm, \mp}$. Hence, we expect SR to occur for $\pi / \Omega_{x} \simeq 1 / \gamma_{ \pm, \mp}$, and, according Eqs. (1) and (2), for

$$
\left(\Gamma_{0}^{\prime}\right)_{\mathrm{SR}}=\frac{9 \sin (\theta) \sqrt{\left|\Delta_{0}^{\prime}\right| \omega_{\mathrm{r}}}}{\pi\left(\overline{\cos ^{2}(2 \pi \hat{x})+\cos ^{2}(2 \pi \hat{y})}\right)} .
$$

For values of the noise strength lower than $\left(\Gamma_{0}^{\prime}\right)_{\mathrm{SR}}$, the optical pumping is too weak to allow the atom to go across the potential barrier every time it reaches a crossing point between the potential curves. On the contrary, for a level of noise much larger than $\left(\Gamma_{0}^{\prime}\right)_{\mathrm{SR}}$, the Hamiltonian motion is broken by the numerous optical pumping cycles so that the atoms cannot follow the driving potential modulation.

Figure 4 clearly shows that the amplitude of the bellshaped curve of $\xi$ versus $\Gamma_{0}^{\prime}$ increases for increasing depths of the driving modulation (i.e. increasing values of $\left.I_{\mathrm{P}} / I_{\mathrm{L}}\right)$. This can be easily explained by the fact that the number of atoms dragged by the moving modulation is greater for deeper driving modulations. We find that the position of the $\mathrm{SR},\left(\Gamma_{0}^{\prime}\right)_{\mathrm{SR}}$, is independent of $I_{\mathrm{P}} / I_{\mathrm{L}}$ in good agreement with the property that Eq. (6) does not involve the driving modulation properties. In fact Eq. (6) only involves the properties of the lattice itself. Note however, that for extreme values of $I_{\mathrm{P}} / I_{\mathrm{L}}$, Eq. (6) certainly no longer works. Indeed, for very weak values of $I_{\mathrm{P}} / I_{\mathrm{L}}$, the propagation modes are not excited and no enhancement of the spatial diffusion can be detected, whereas for very high values, the lattice is too much perturbated to influence the dynamics of the atoms.

In order to check the validity of the theoretical synchronization condition (6), we determined the enhancement factor $\xi$ versus the noise strength $\Gamma_{0}^{\prime}$ for various fixed values of the lattice potential depth $\Delta_{0}^{\prime}$. We found results as in Fig. 4 and we consequently determined the position of the SR, $\left(\Gamma_{0}^{\prime}\right)_{\mathrm{SR}}$, as a function of $\Delta_{0}^{\prime}$ in a wide range. We plot our results in Fig. [5] together with the theoretical curve corresponding to Eq. (6) for a 2D lattice $(\hat{y}=0)$ where $\overline{\cos ^{2}(2 \pi \hat{x})+\cos ^{2}(2 \pi \hat{y})}=3 / 2$ as in the simulations. Note that no free parameter is used to fit the numerical data.

We obtain an excellent qualitative as well as quantitative agreement between the numerical results and the theory. We thus conclude that the resonance observed in Fig. 4 corresponds to the phenomenon of SR for which the dissipative processes (optical pumping) are synchronized with the Hamiltonian motion (half-oscillations in the potential wells) of the atoms in a propagation mode. Similar results are expected in a full $3 \mathrm{D}$ situation. In that case, the scaling factor of $\left(\Gamma_{0}^{\prime}\right)_{\mathrm{SR}}$ versus $\sqrt{\left|\Delta_{0}^{\prime}\right|}$ is however expected to be slightly different because of a different average value for $\overline{\cos ^{2}(2 \pi \hat{x})+\cos ^{2}(2 \pi \hat{y})}$. In the $x$ direction, a uniform average is valid because the atoms excited in the propagation modes are moving along $x$ but the average over $y$ should take into account the atomic localization in the lattice [19]. In fact, because the potential minima 


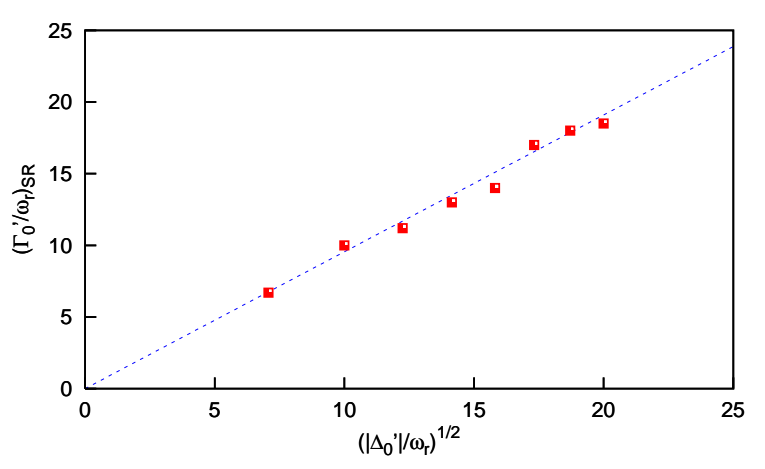

FIG. 5: Position of the stochastic resonance versus the square root of the modulation depth and comparison to the theoretical value given by Eq. (6). Parameters for the simulations are $\theta=30^{\circ}$ and $I_{\mathrm{P}} / I_{\mathrm{L}}=9 \%$.

correspond to $\hat{y} \equiv 0[\pi]$, we expect $\overline{\cos ^{2}(2 \pi \hat{x})+\cos ^{2}(2 \pi \hat{y})}$ not to be very different from $3 / 2$.

\section{MICROSCOPIC DYNAMICS AND STOCHASTIC RESONANCE}

\section{A. Atomic bunching in a moving frame}

In section III] we have given evidences for the realization of SR in the enhancement of the spatial diffusion of the atomic cloud in the $x$ - direction. However, no detailed study of the microscopic behavior of the atoms in the presence of a variable amount of noise has been performed so far. In particular, the problem arises as to give a direct evidence that the propagation modes are really excited resonantly for a given amount of noise. In order to adress this problem, we shall directly determine the atomic population in a propagation mode versus $\Gamma_{0}^{\prime}$. In the configuration depicted in Fig. 1 two potential modulations with a spatial period $\lambda_{\text {mod }}$ and a velocity $v_{\text {mod }}$, dragg the atoms symmetrically with respect to axis $(O z)$ in the directions $\vec{u}^{+}$and $\vec{u}^{-}$. We therefore expect that the atomic distribution is spatially modulated in the moving potential modulations and particularly that the atoms are bunched around the moving potential minima.

We determined the atomic spatial distribution $N^{ \pm}(u)$ in a frame moving in the direction and at the velocity of each mode. Here $u$ is the space coordinate in direction $\vec{u}^{ \pm}$. Because of the low atomic filling rates in dissipative optical lattices, $N^{ \pm}(u)$ is in fact calculated by accumulating over time. Moreover, we cut the whole space

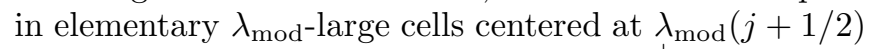
and we add the populations of each cell $N_{j}^{ \pm}\left(u+j \lambda_{\text {mod }}\right)$, with $u \in\left[0, \lambda_{\text {mod }}\left[\right.\right.$, so that $N^{ \pm}(u)=\sum_{j} N_{j}^{ \pm}\left(u+j \lambda_{\text {mod }}\right)$. $N^{ \pm}(u)$ results from the superimposition of several terms corresponding to atoms with different dynamical behaviors. On the one hand, the atoms trapped in the lattice sites and the atoms dragged along the moving potential modulation $\vec{u}^{\mp}$ have a modulated and stationary spatial distribution respectively in the un-moving frame and in the frame moving as the mode $\vec{u}^{\mp}$. Because these frames move with respect to our reference frame (attached to the mode $\vec{u}^{ \pm}$), their contribution is uniform with respect to $u$ and contributes as a constant number. On the other hand, the atoms dragged along the potential modulation $\vec{u}^{ \pm}$are approximately at rest in our frame and consequently give the shape of moving matter modulation along the mode $\vec{u}^{ \pm}$. In a linear regime for the atomic response to the dragging, valid for low enough values of $I_{\mathrm{L}} / I_{\mathrm{P}}$, we expect the contribution to $N^{ \pm}(u)$ of the atoms from mode $\vec{u}^{ \pm}$to be sinusoidal as the potential modulation itself. We thus conclude that the atomic spatial distribution in the moving frame should be

$$
N^{ \pm}(u)=C\left[1+A \sin \left(\frac{2 \pi u}{\lambda_{\bmod }}+\varphi\right)\right] .
$$

Here the amplitude $A$ characterizes the fraction of atoms in the propagation mode $\vec{u}^{ \pm}$and the phase $\varphi$ accounts for the delay of the matter modulation with respect to the dragging potential modulation due to the finite response time of the atoms in the lattice. $C=\int_{0}^{\lambda_{\bmod }} \frac{d u}{\lambda_{\bmod }} N^{ \pm}(u)$ is determined by the total number of atoms used in the simulations and the integration time window. $C$ is thus invariant when changing the lattice parameters.

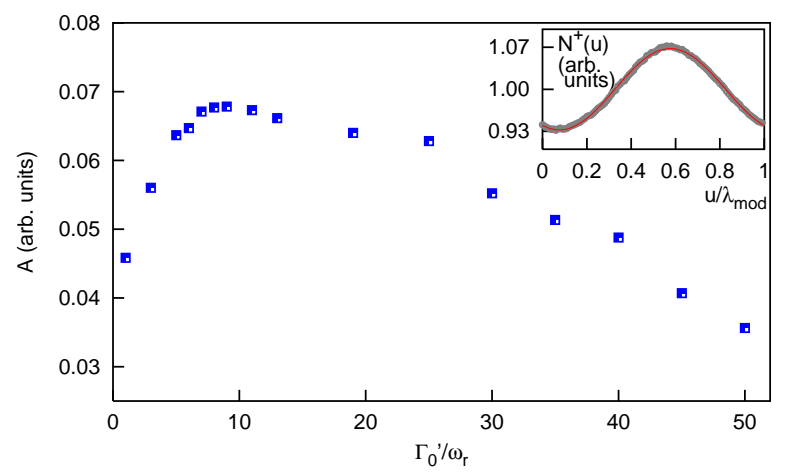

FIG. 6: Amplitude of the atomic fraction in the propagation mode versus the optical pumping rate. The phenomenon of stochastic resonance is clearly visible. Parameters for the simulations are $\Delta_{0}^{\prime}=-50 \omega_{\mathrm{r}}, \theta=30^{\circ}$ and $I_{\mathrm{P}} / I_{\mathrm{L}}=9 \%$. Inset: Numerical result for the atomic bunching in the moving frame corresponding to the propagation mode together with a sinusoidal fit of the form of Eq. (7). Here $\Gamma_{0}^{\prime}=9 \omega_{\mathrm{r}}$.

A typical result for $N^{+}(u)$ is plotted in the inset of Fig. [6 together with a fit to a function of the form of Eq. (7) where $A$ and $\varphi$ are the free parameters. The numerical results are found to be in excellent agreement with Eq. (7). We plot in Fig. [6 the amplitude $A$ of the sinusoidal component in $N^{+}(u)$ as a function of the optical pumping rate $\Gamma_{0}^{\prime}$. We obtain a bell-shaped curve which is characteristic of SR. This result constitutes the first direct evidence on a microscopic observable that the Brillouin-like propagation modes are resonantly excited for a given level of dissipative effects. The position of the resonance $\left(\Gamma_{0}^{\prime}\right)_{\mathrm{SR}}$ is also in good agreement with the 
results plotted in Fig. 5 and corresponding to the SR observed in the enhancement factor of the spatial diffusion coefficient along the direction $x$. These results confirm by a direct method that the Brillouin propagation modes are excited resonantly when the synchronization condition (66) between the Hamiltonian motion and the dissipative processes in the optical lattice is fulfilled.

\section{B. Brillouin spectroscopy}

A very powerful and widely used technique to probe the dynamical properties of a cold atomic sample in an optical lattice is nonlinear pump-probe spectroscopy 7, 21, 27 and we adress now the problem to known whether SR can be observed in spectroscopy experiments. The method consists in using the laser $(P)$ as the probe and the lattice beams $\left(L_{ \pm \mu}\right)$ as the pump (see Fig. 1). As mentionned previously, the interference between the probe and pump beams induces moving modulations of the laser field which create time-dependent modulations of various observables in the medium (spatial and velocity distributions, polarization, etc ...) with a non-zero phase with respect to the laser field modulation. This is connected to the finite response time of the atoms in the lattice. The diffraction of the pump beams onto the matter modulation induces a modification of the probe intensity $I$ which is measured as a function of the detuning between the probe and the pump beams $\delta$ [28]. The elementary excitation modes of the atomic sample appear in the spectrum ( $I$ as a function of $\delta$ ) as resonances whose positions and widths are related respectively to the real part and to the imaginary part of the eigenfrequency of the mode [29, 30]. It is thus clear that the amplitude of the excitation resonance in the spectrum depends on both the amplitude and the phase of the observable modulation involved in the light scattering process. For a $3 \mathrm{D}$-lin $\perp$ lin configuration with a probe beam aligned along $(\mathrm{Oz})$, the spectrum consists of three kinds of resonances: first, the Rayleigh lines which relate to the pure relaxation processes associated to spatial diffusion and damping of the kinetic energy [31, 32]; second, the sideband Raman lines which correspond to transitions between vibrational bound states in the potential wells 27]; third, the Brillouin resonances which correspond to the propagation modes discussed in section [1] 20].

In the case of the Brillouin line, the amplitude of the resonance is closely related to the atomic spatial distribution in the moving reference frame and particularly to the values of $A$ and $\varphi$. In fact, we observed in the numerical simulations that $\varphi$ weakly depends on $\Gamma_{0}^{\prime}$ for a fixed value of $\Delta_{0}^{\prime}$ in the range of parameters considered here (the range of values for $\varphi$ is found to be $\Delta \varphi / \pi<10 \%$ ). Hence, the amplitude of the Brillouin resonance should characterize the amplitude $A$ of the moving matter modulation and should therefore show a SR behavior.

We performed numerical simulations of the pumpprobe spectra in the lattice for different values of the optical pumping rate $\Gamma_{0}^{\prime}$ and of the light shift per beam $\Delta_{0}^{\prime}$ by means of a method equivalent to the one described in Ref. 28]. In order to determine the amplitude of the Brillouin resonance, we fit the spectra to functions of the form

$$
\begin{array}{r}
I(\delta)=A_{\mathrm{e}} \delta+B_{\mathrm{e}}+A_{\mathrm{R}} \exp \left[-\frac{\left(\delta-\Omega_{\mathrm{R}}\right)^{2}}{2 \sigma_{\mathrm{R}}^{2}}\right]+ \\
+A_{\mathrm{B}} \exp \left[-\frac{\left(\delta-\Omega_{\mathrm{B}}\right)^{2}}{2 \sigma_{\mathrm{B}}^{2}}\right]
\end{array}
$$

with $A_{\mathrm{e}}, B_{\mathrm{e}}, A_{\mathrm{R}}, \sigma_{\mathrm{R}}, A_{\mathrm{B}}$ and $\sigma_{\mathrm{B}}$ as free parameters. The linear term in Eq. (81) accounts for the very large central Rayleigh resonance [32] in a restricted range for $\delta$ close to the Brillouin resonance, whereas the two Gaussian functions account for the Raman (R) and Brillouin (B) lines centered at $\Omega_{\mathrm{R}, \mathrm{B}}$ and with a half-width at $1 / \sqrt{e}$ equal to $\sigma_{\mathrm{R}, \mathrm{B}}$. The a priori justification of the Gaussian shapes of the Raman and Brillouin lines is out of the scope of this paper and the approximation of the spectra to Eq. (8) is validated by the fact that it fits well the numerical spectra as shown in the inset of Fig. 7

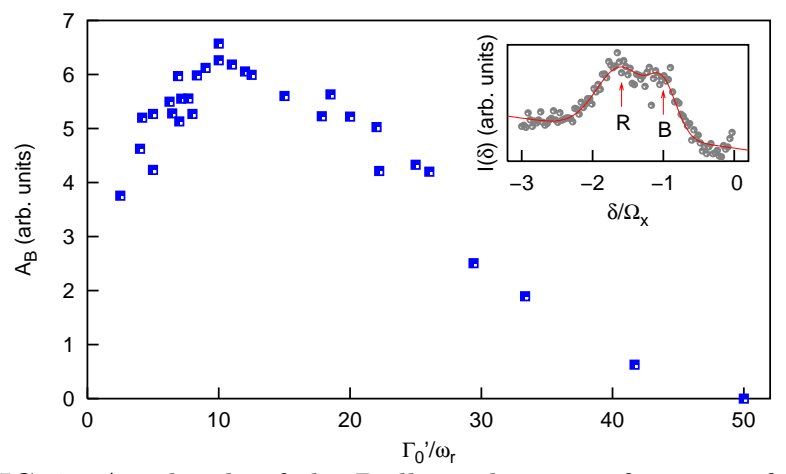

FIG. 7: Amplitude of the Brillouin line as a function of the optical pumping rate for a fixed potential depth as obtained from nonlinear spectroscopy simulations. Parameters for the simulations are the same as in Fig. 6] Inset: Numerical result of a nonlinear spectrum $\left(\Gamma_{0}^{\prime}=-5.55 \omega_{\mathrm{r}}\right)$; the Raman $(\mathrm{R})$ and Brillouin (B) resonances are indicated on the plot.

We finally plot in Fig. 7 the amplitude of the Brillouin resonance $A_{\mathrm{B}}$ as a function of the optical pumping rate $\Gamma_{0}^{\prime}$ for a fixed value of the light shift per beam $\Delta_{0}^{\prime}$. Again, we find a bell-shaped curve which corresponds to $\mathrm{SR}$. The position of the resonance, $\left(\Gamma_{0}^{\prime}\right)_{\mathrm{SR}}$, is found to be in good agreement with the position observed for the enhancement of spatial diffusion (section IIII) and for the spatial distribution in moving frames (section IVA). We therefore conclude that the spectroscopy methods provide a new means for observing $\mathrm{SR}$ on the propagation modes in a dissipative optical lattice.

\section{CONCLUSION}

In conclusion, we have performed a detailed theoretical study of the influence of the noise related to the dis- 
sipative processes (optical pumping) on the excitation process of the Brillouin propagation modes in an optical lattice. Our results are mainly obtained from semiclassical Monte-Carlo simulations. We have shown that there is a specific amount of noise for which the excitation of the modes is resonant. This corresponds to the phenomenon of stochastic resonance (SR), obtained when the Hamiltonian motion of an atom in the light potential and the dissipative processes in the lattice are precisely synchronized. This result can be understood from the fact that the excitation of a mode involves synchronized half-oscillations in the potential wells and optical pumping cycles. We have given a direct evidence for SR by means of the bunching properties in frames moving in the direction and at the same velocity as the propagation modes, and an indirect evidence by the observation of the resonant enhancement of spatial diffusion. We have shown that the resonant excitation of the modes, and thus SR, can be observed by means of pump-probe spectroscopy. The three methods give results for the dependence of the SR point versus the lattice parameters in good agreement. We have also derived a simple analytical model for the synchronization condition in excellent qualitative as well as quantitative agreement with the results of our numerical simulations.

The system that we examined in this paper is thus a good candidate for the experimental realization of SR in a system where the relevant parameters for Hamiltonian motion and dissipative effects can vary independently and that can be probed easily. Note also that optical lattices can be designed in a large variety of configurations [10]. This system may thus model many different physical situations. First experimental results have been already obtained in a variant of the configuration consid- ered in this paper [33]. In Ref. [33], Schiavoni et al show that the excitation of the Brillouin propagation modes is nonmonotonic versus the noise strength. Our modelsystem is known to correctly simulate the real experimental situations. We therefore expect our results to be reproductible in current experiments. In particular, it would be interesting to investigate the atomic bunching in moving frames in order to show the coherent collective excitation aspect of the propagation modes. This may be achieved via Bragg scattering, a technique that has been already used to give evidence of atomic localization in optical lattices [34]. The characterization of SR via pump-probe spectroscopy experiments, a technique wellmastered in experiments on cold atoms [7, 21], would also give interesting results. At last, an experimental investigation of the lattice parameters dependence of the SR point $\left(\left(\Gamma_{0}^{\prime}\right)_{\mathrm{SR}}\right)$ would give interesting insights onto the excitation process of the Brillouin propagation modes in dissipative optical lattices.

\section{ACKNOWLEDGMENTS}

We are grateful to Anders Kastberg for useful comments on the paper. We also thank Ferruccio Renzoni and Michele Schiavoni for various discussions. This work was supported by CNRS, the European Commission (TMR network "Quantum Structures", contract FMRXCT96-0077) and by Région Ile de France under contract E.1220. Laboratoire Kastler Brossel is an "unité mixte de recherche de l'Ecole Normale Supérieure et de l'Université Pierre et Marie Curie associée au Centre National de la Recherche Scientifique (CNRS)".
[1] S. Chu, Nobel Lectures, Rev. Mod. Phys. 70, 685 (1998); C. Cohen-Tannoudji, Nobel Lectures, ibid. 70, 707 (1998); W. D. Phillips, Nobel Lectures, ibid. 70, 721 (1998).

[2] E. A. Cornell and C. E. Wieman, Nobel Lectures, Rev. Mod. Phys. 74, 875 (2002); W. Ketterle, Nobel Lectures, ibid. 74, 1131 (2002).

[3] B. DeMarco and D. S. Jin, Science 285, 1703 (1999); A. G. Truscott, K. E. Strecker, W. I. McAlexander, G. B. Partridge and R. G. Hulet, Science 291, 2570 (2001).

[4] Special issue "Quantum transport of atoms in optical lattices", J. Opt. B: Quantum Semiclass. Opt. 2, M. Raizen and W. Schleich eds., 589 (2000).

[5] J. Dalibard and C. Cohen-Tannoudji, J. Opt. Soc. Am. B 6, 2023 (1989); P. J. Ungar, D. S. Weiss, E. Riis and S. Chu, ibid. 6, 2058 (1989).

[6] Y. Castin and J. Dalibard, Europhys. Lett. 14, 761 (1991).

[7] P. Verkerk, B. Lounis, C. Salomon, C. Cohen-Tannoudji, J.-Y. Courtois and G. Grynberg, Phys. Rev. Lett. 68, 3861 (1992).

[8] G. Grynberg, B. Lounis, P. Verkerk, J.-Y. Courtois and C. Salomon, Phys. Rev. Lett. 68, 3861 (1992); M. Gatzke, G. Birkl, P. S. Jessen, A. Kastberg, S. L. Rolston and W. D. Phillips, Phys. Rev. A 55, R3987 (1997).

[9] J. S. Jessen and I. H. Deutsch, Adv. At. Mol. Opt. Phys. 37, 95 (1996).

[10] G. Grynberg and C. Mennerat-Robilliard, Phys. Rep. 355, 335 (2001).

[11] C. Cohen-Tannoudji, Les Houches summer school of theoretical physics 1990, Session LIII, in "Fundamental systems in Quantum Optics", J. Dalibard, J. M. Raimond and J. Zinn-Justin eds., North Holland, Amsterdam, Elsevier Science Publishers B.V., (1991).

[12] Chaos and noise in biology and medicine, Proceedings of the International School of Biophysics Casamicciola 1997, M. Barbi and S. Chillemi eds., World Scientific Publishing, Singapore (1998).

[13] C. Mennerat-Robilliard, D. Lucas, S. Guibal, J. Tabosa, C. Jurczak, J.-Y. Courtois and G. Grynberg, Phys. Rev. Lett. 82, 851 (1999); C. Mennerat-Robilliard, D. Lucas and G. Grynberg, Appl. Phys. A 75, 213 (2002).

[14] M. Schiavoni, L. Sanchez-Palencia, F. Renzoni and G. Grynberg, Phys. Rev. Lett. 90, 094101 (2003). 
[15] L. Sanchez-Palencia, F.-R. Carminati, M. Schiavoni, F. Renzoni and G. Grynberg, Phys. Rev. Lett. 88, 133903 (2002).

[16] L. Gammaitoni, P. Hänggi, P. Jung and F. Marchesoni, Rev. Mod. Phys. 70, 223 (1998); L. Fronzoni and R. Mannella, J. Stat. Phys. 70, 501 (1993); R. Löfstedt and S. N. Coppersmith, Phys. Rev. Lett. 72, 1947 (1994); M. I. Dykman, D. G. Luchinsky, R. Mannella, P. V. E. McClintock, N. D. Stein and N. G. Stocks, J. Stat. Phys. 70, 479 (1993).

[17] K. I. Petsas, A. B. Coates and G. Grynberg, Phys. Rev. A 50, 5173 (1994); A. Kastberg, W. D. Phillips, S. L. Rolston, R. J. C. Spreeuw and P. S. Jessen, Phys. Rev. Lett. 74, 1542 (1995).

[18] Y. Castin, K. Berg-Sørensen, J. Dalibard and K. Mølmer, Phys. Rev. A 50, 5092 (1994); J. Jersblad, H. Ellmann, L. Sanchez-Palencia and A. Kastberg, Eur. Phys. J. D 22, 333 (2003).

[19] L. Sanchez-Palencia, P. Horak and G. Grynberg, Eur. Phys. J. D 18, 353 (2002).

[20] J.-Y. Courtois, S. Guibal, D. R. Meacher, P. Verkerk and G. Grynberg, Phys. Rev. Lett. 77, 40 (1996).

[21] A. Hemmerich and T. W. Hänsch, Phys. Rev. Lett. 70, 410 (1993).

[22] S. Marksteiner, K. Ellinger and P. Zoller, Phys. Rev. A 53, 3409 (1996).

[23] E. P. Wigner, Phys. Rev. 40, 749 (1932).

[24] K. I. Petsas, G. Grynberg and J.Y. Courtois, Eur. Phys. J. D 6, 29 (1999).

[25] H. Risken, The Fokker-Planck equation, Springer, Berlin (1989).

[26] C. Itzykson and J. M. Drouffe, Statistical Field Theory vol. 1: From Brownian Motion to Renormalization and Lattice Gauge Theory, Cambridge University Press, Cambridge (1991).

[27] J.-Y. Courtois and G. Grynberg, Phys. Rev. A 46, 7060 (1992); J.-Y. Courtois, Ph-D dissertation (1993) and Ann. Phys. Fr. 21, 1 (1996).

[28] J.-Y. Courtois, Proceedings of the International school of Physics "Enrico Fermi", course CXXXI, A. Aspect, W. Barletta and R. Bonifacio eds., IOS Press, Amsterdam (1996).

[29] Y. R. Shen, The principles of nonlinear optics, Wiley, New York (1984); R. W. Boyd, Nonlinear optics, Academic Press, New York (1992).

[30] J.-Y. Courtois and G. Grynberg, Adv. At. Mol. Opt. Phys. 36, 87 (1996).

[31] C. Jurczak, B. Desruelle, K. Sengstock, J.-Y. Courtois, C. I. Westbrook and A. Aspect, Phys. Rev. Lett. 77, 1727 (1996); S. Guibal, C. Mennerat-Robilliard, D. Larousserie, C. Triché, J.-Y. Courtois and G. Grynberg, Phys. Rev. Lett. 78, 4709 (1997).

[32] F.-R. Carminati, L. Sanchez-Palencia, M. Schiavoni, F. Renzoni and G. Grynberg, Phys. Rev. Lett. 90, 043901 (2003).

[33] M. Schiavoni, L. Sanchez-Palencia, F.-R. Carminati, F. Renzoni and G. Grynberg, Europhys. Lett. 59, 493 (2002).

[34] G. Birkl, M. Gatzke, I. H. Deutsch, S. L. Rolston and W. D. Phillips, Phys. Rev. Lett. 75, 2823 (1995); M. Weidemuller, A. Hemmerich, A. Görlitz, T. Esslinger and T. W. Hänsch, Phys. Rev. Lett. 75, 4583 (1995).

[35] Here, $\hat{\mu}=\mu / \lambda_{\mu}$, where $\lambda_{\mu}$ is the lattice spacing in direction $\mu \in\{x, y, z\}$. For the $3 \mathrm{D}$-lin $\perp$ lin configuration, $\lambda_{x, y}=\lambda /(k \sin (\theta))$ and $\lambda_{z}=\lambda /(2 k \cos (\theta))$. 